\title{
DYNAMICS OF A STOCHASTIC POPULATION MODEL WITH ALLEE EFFECT AND JUMPS
}

\author{
RONG $\operatorname{LiU}^{1}$ (D) AND GUIRONG $\mathrm{LiU}^{2, *}$ (B)
}

\begin{abstract}
This paper is concerned with a stochastic population model with Allee effect and jumps. First, we show the global existence of almost surely positive solution to the model. Next, exponential extinction and persistence in mean are discussed. Then, we investigated the global attractivity and stability in distribution. At last, some numerical results are given. The results show that if attack rate $a$ is in the intermediate range or very large, the population will go extinct. Under the premise that attack rate $a$ is less than growth rate $r$, if the noise intensity or jump is relatively large, the population will become extinct; on the contrary, the population will be persistent in mean. The results in this paper generalize and improve the previous related results.
\end{abstract}

Mathematics Subject Classification. 60H10, 60J60, 92D25, 92D40.

Received August 21, 2021. Accepted January 10, 2022.

\section{INTRODUCTION}

The Allee effect represents the relationship between population growth and population density. That is, for a population with Allee effect, if its population density is too sparse, it is so difficult to find a mate that reproduction does not compensate for mortality, then its population number will be reduced (see $[1,10,34]$ ). Allee effects mainly classified into two broad categories: strong Allee effect and weak Allee effect (see [4]). There is a threshold population level for the strong Allee effect such that the species become extinct below this threshold population density. On the other hand, the weak Allee effect occurs when the growth rate reduces but remains positive at low population density (see [4]). Recently, many literatures have studied the population dynamics with Allee effects (see [5-7, 14]). In [7], the author used Poincaré-Bendixson theory to study the following single population model with Allee effect

$$
x^{\prime}(t)=x(t)\left[r-c x(t)-\frac{a}{1+h a x(t)}\right]
$$

with $x(0)=x_{0}$. Here, $r$ represents the intrinsic growth rate, $c$ stands for the strength of intra-competition of the population. Under predation satiation circumstance, $h$ and $a$ represent the handling time and the attack rate of predator, respectively. Here, $\frac{a x}{1+h a x}$ is the Allee effect (see [10] for more details).

Keywords and phrases: Lévy noise, Allee effect, extinction, attractivity, stability.

${ }^{1}$ School of Applied Mathematics, Shanxi University of Finance and Economics, Taiyuan, Shanxi 030006, PR China.

2 School of Mathematical Sciences, Shanxi University, Taiyuan, Shanxi 030006, PR China.

* Corresponding author: lgr5791@sxu.edu.cn

(C) The authors. Published by EDP Sciences, 2022 
In addition, populations are actually subject to the environmental noise. From [21], the extra noise can be divided into white noise and color noise. The white noise is uncorrelated, that is, each and every randomly drawn noise value is totally independent on previous value. However, the colored noise is temporally autocorrelated, that is, the values of random numbers used in the noise process will depend on the previous ones. Thus, it is more objective to modeling stochastic population models with white noise in mathematical biology (to name a few, see $[8,9,11,22,25,30])$. In these papers the authors revealed how noise affects the population dynamics. Especially, $[8,9,11,25,30]$ investigated the dynamics of stochastic population models with Allee effect. There are many different ways to introduce environmental noise into the model. For example, due to the environment's continuous fluctuation, the intrinsic growth rate $r$ always fluctuates around some average value. In this sense $r \rightarrow r+\sigma \dot{B}(t)$. Here $\sigma^{2}$ represents the intensity of white noise $\dot{B}(t)$. Thus, one can get the following stochastic population model with Allee effect

$$
\mathrm{d} x(t)=x(t)\left[r-c x(t)-\frac{a}{1+h a x(t)}\right] \mathrm{d} t+\sigma x(t) \mathrm{d} B(t)
$$

with $x(0)=x_{0}>0$.

As we all know, Brownian motion is a stochastic process whose paths are continuous. However, natural populations are actually subject to sudden environmental shocks (earthquakes, hurricanes, epidemics, etc.), which have to be taken into account when the population dynamics model is established. Here, a sudden environmental shock will cause a sudden shift on the size of biological population, and the mathematical explanation is that sample paths are not continuous almost surely. It is recognized that stochastic differential equations with Lévy process are quite suitable to describe such discontinuous system. From [32], Lévy processes are stochastic processes with stationary and independent increments, in which they can form some special classes of both semi-martingales and Markov processes. Moreover, Lévy processes are not continuous, but their sample paths are right-continuous and have a number of random jump discontinuities occurring at random times, on each finite time interval. Thus, in order to depict these sudden changes in the environment, as in [17, 18, 27-29], we propose to introduce Lévy process into the stochastic model (1.2) and obtain the following stochastic population model with Lévy noise and Allee effect

$$
\mathrm{d} x(t)=x(t-)\left[r-c x(t-)-\frac{a}{1+h a x(t-)}\right] \mathrm{d} t+\sigma x(t-) \mathrm{d} B(t)+\int_{\Gamma} x(t-) \gamma(z) \tilde{N}(\mathrm{~d} t, \mathrm{~d} z)
$$

with $x(0)=x_{0}>0$. Here $x(t-)$ means the left limit of $x(t)$, the parameters $r, c, a$ and $h$ are defined as in model (1.1). $B(t)$ is a standard Brownian motion defined on a filtered compete probability space $\left(\Omega, \mathcal{F},\left\{\mathcal{F}_{t}\right\}_{t \geq 0}, \mathbb{P}\right)$ satisfying usual hypotheses. $N(\cdot, \cdot)$ is a Poisson process with parameter $\lambda$ on the measurable subset $\Gamma \subset[0, \infty)$ with $\lambda(\Gamma)<\infty$. The compensated Poisson random measure $\widetilde{N}(\mathrm{~d} t, \mathrm{~d} z)=N(\mathrm{~d} t, \mathrm{~d} z)-\lambda(\mathrm{d} z) \mathrm{d} t$ is a martingale, which is independent of $\dot{B}(t) . \sigma^{2}$ represents the intensity of $\dot{B}(t) . \gamma$ is the effect of jumps on the population.

The remainder of this paper is organized as follows. In Section 2, we show that the model has a unique global positive solution by the comparison theorem of stochastic differential equations. In Section 3, the asymptotic pathwise behavior and stochastically ultimate boundedness of the model are investigated. In Section 4, We discuss the effect of Allee effect and stochastic perturbation on population dynamic behavior. Further, in Section 5, we first show that the solution of the model is globally attractive in mean. Then, the stability in distribution of the model is investigated. Section 6 contains numerical results, which are used to demonstrate the effectiveness of the theoretical results in this paper. The paper ends with a conclusion. The results in this paper generalize and improve the previous related results.

Throughout this paper, set $\mathbb{R}_{+}=(0, \infty), \overline{\mathbb{R}}_{+}=[0, \infty)$ and assume that $\gamma(z)>-1$ is a bounded function. From the boundedness of $\gamma(z)$, there is $K>0$ satisfying

$$
\int_{\Gamma}[\ln (1+\gamma(z))]^{2} \lambda(\mathrm{d} z)<K
$$


For simplicity, let $M(t)=\int_{0}^{t} \int_{\Gamma} \ln (1+\gamma(z)) \tilde{N}(\mathrm{~d} s, \mathrm{~d} z)$ and $\beta=\int_{\Gamma}[\ln (1+\gamma(z))-\gamma(z)] \lambda(\mathrm{d} z)-\frac{\sigma^{2}}{2}$. Note that $1-s+\ln s \leq 0$ for $s>0$, from which it follows that $\beta=\int_{\Gamma}[\ln (1+\gamma(z))-(1+\gamma(z))+1] \lambda(\mathrm{d} z)-\frac{\sigma^{2}}{2} \leq-\frac{\sigma^{2}}{2}<0$.

\section{Existence AND UNIQUENESS OF THE POSITIVE SOLUTION}

Note that $x(t)$ is the size of the population. Thus, we should consider positive global solutions of (1.3).

Theorem 2.1. For any initial value $x_{0} \in \mathbb{R}_{+}$, model (1.3) has a unique global positive solution $x(t)$ for all $t \geq 0$ with probability one, namely,

$$
\mathbb{P}\{x(t)>0: \text { for any } t \geq 0\}=1 \text {. }
$$

Proof. Consider the following stochastic differential equation

$$
\mathrm{d} v(t)=\left[r-c e^{v(t)}-\frac{a}{1+h a e^{v(t)}}+\beta\right] \mathrm{d} t+\sigma \mathrm{d} B(t)+\int_{\Gamma} \ln (1+\gamma(z)) \tilde{N}(\mathrm{~d} t, \mathrm{~d} z)
$$

with $v(0)=\ln x_{0}$. Note that the coefficients of (2.1) satisfy the local Lipschitz condition. Hence, (2.1) has a unique maximal local solution $v(t)$ on $\left[0, \tau_{e}\right)$, where $\tau_{e}$ is the explosion time. Let $x(t)=e^{v(t)}$. From the generalized Itô formula, model (1.3) has a unique positive local solution $x(t)=e^{v(t)}$ on $\left[0, \tau_{e}\right)$ satisfying $x(0)=x_{0}$.

Next, we show that $v(t)$ is a global solution to equation $(2.1)$, i.e. $\tau_{e}=\infty$. Consider the following two stochastic auxiliary systems

$$
\begin{aligned}
& \left\{\begin{array}{l}
\mathrm{d} \phi(t)=\phi(t-)[r-c \phi(t-)] \mathrm{d} t+\sigma \phi(t-) \mathrm{d} B(t)+\int_{\Gamma} \phi(t-) \gamma(z) \tilde{N}(\mathrm{~d} t, \mathrm{~d} z), \\
\phi(0)=x_{0}
\end{array}\right. \\
& \left\{\begin{array}{l}
\mathrm{d} \psi(t)=\psi(t-)[(r-a)-c \psi(t-)] \mathrm{d} t+\sigma \psi(t-) \mathrm{d} B(t)+\int_{\Gamma} \psi(t-) \gamma(z) \tilde{N}(\mathrm{~d} t, \mathrm{~d} z), \\
\psi(0)=x_{0}
\end{array}\right.
\end{aligned}
$$

From Lemma 4.2 in [2], for any $\phi(0)=x_{0}$, equation (2.2) has a unique solution $\phi(t)>0$ on $\mathbb{R}_{+}$and can be explicitly solved as follow

$$
\phi(t)=\frac{\exp \{(r+\beta) t+\sigma B(t)+M(t)\}}{\frac{1}{x_{0}}+c \int_{0}^{t} \exp \{(r+\beta) s+\sigma B(s)+M(s)\} \mathrm{d} s} .
$$

Similarly, for any $\psi(0)=x_{0}$, equation (2.3) has a unique solution $\psi(t)>0$ on $\mathbb{R}_{+}$and

$$
\psi(t)=\frac{\exp \{(r-a+\beta) t+\sigma B(t)+M(t)\}}{\frac{1}{x_{0}}+c \int_{0}^{t} \exp \{(r-a+\beta) s+\sigma B(s)+M(s)\} \mathrm{d} s} .
$$

From stochastic comparison theorem ([20], Thm. 3.1), it follows that

$$
0<\psi(t) \leq x(t) \leq \phi(t), \text { a.s., for } t \in\left[0, \tau_{e}\right)
$$

Further, we have

$$
\ln \psi(t) \leq v(t) \leq \ln \phi(t), \text { a.s., for } t \in\left[0, \tau_{e}\right)
$$


Since $\ln \phi(t)$ and $\ln \psi(t)$ exist on $[0, \infty)$, it follows that $\tau_{e}=\infty$. Thus, for any initial value $v(0)=\ln x_{0}$, equation (2.1) has a unique solution $v(t)$ on $\mathbb{R}_{+}$a.s. Therefore, for any initial value $x_{0} \in \mathbb{R}_{+}$, model (1.3) has a unique solution $x(t)>0$ on $\mathbb{R}_{+}$a.s. The proof is therefore complete.

\section{Asymptotic properties of the solution}

In this section, by using the generalized exponential martingale inequality and Borel-Cantelli lemma, we investigate the asymptotic pathwise behavior of the model. Then, we continue to examine the stochastically ultimate boundedness of the model.

Lemma 3.1 (see $[16,24])$. Let $f:[0, \infty) \rightarrow \mathbb{R}$ and $h:[0, \infty) \times \Gamma \rightarrow \mathbb{R}$ be both predictable $\left\{\mathcal{F}_{t}\right\}$-adapted processes such that for any $T>0$,

$$
\int_{0}^{T}|f(t)|^{2} \mathrm{~d} t<\infty \text { a.s. and } \int_{0}^{T} \int_{\Gamma}|h(t, z)|^{2} \lambda(\mathrm{d} z) \mathrm{d} t<\infty \text { a.s. }
$$

Then, for any positive constants $\vartheta, \varrho$,

$$
\mathbb{P}\left\{\sup _{0 \leq t \leq T}[A(t)-\Phi(t)]>\varrho\right\} \leq e^{-\vartheta \varrho} .
$$

Here $A(t)=\int_{0}^{t} f(s) \mathrm{d} B(s)+\int_{0}^{t} \int_{\Gamma} h(s, z) \tilde{N}(\mathrm{~d} s, \mathrm{~d} z), \Phi(t)=\frac{\vartheta}{2} \int_{0}^{t}|f(s)|^{2} \mathrm{~d} s+\frac{1}{\vartheta} \int_{0}^{t} \int_{\Gamma}\left[e^{\vartheta h(s, z)}-1-\vartheta h(s, z)\right] \lambda(\mathrm{d} z) \mathrm{d} s$.

Theorem 3.2. For any $x_{0} \in \mathbb{R}_{+}$, let $x(t)$ be the solution of model (1.3) with initial value $x(0)=x_{0}$. Then

$$
\limsup _{t \rightarrow \infty} \frac{\ln x(t)}{\ln t} \leq 1 \text { a.s. }
$$

Proof. Applying Itôs formula to $e^{t} \ln x$ and using inequality $\ln x \leq x-1$ for $x>0$, we have

$$
\begin{aligned}
e^{t} \ln x(t)= & \ln x_{0}+\int_{0}^{t} e^{s}\left[\ln x(s)+r-c x(s)-\frac{a}{1+h a x(s)}\right] \mathrm{d} s-\frac{1}{2} \int_{0}^{t} \sigma^{2} e^{s} \mathrm{~d} s \\
& +\int_{0}^{t} \int_{\Gamma} e^{s}[\ln (1+\gamma(z))-\gamma(z)] \lambda(\mathrm{d} z) \mathrm{d} s-\int_{0}^{t} \sigma e^{s} \mathrm{~d} B(s) \\
& +\int_{0}^{t} \int_{\Gamma} e^{s} \ln (1+\gamma(z)) \tilde{N}(\mathrm{~d} s, \mathrm{~d} z) \\
\leq & \ln x_{0}+\int_{0}^{t} e^{s}\left[\ln x(s)+r-c x(s)-\frac{a}{1+h a x(s)}\right] \mathrm{d} s-\frac{1}{2} \int_{0}^{t} \sigma^{2} e^{s} \mathrm{~d} s \\
& +\int_{0}^{t}-\sigma e^{s} \mathrm{~d} B(s)+\int_{0}^{t} \int_{\Gamma} e^{s} \ln (1+\gamma(z)) \tilde{N}(\mathrm{~d} s, \mathrm{~d} z) .
\end{aligned}
$$

Note that $\int_{0}^{T} \sigma^{2} e^{2 s} \mathrm{~d} s=\frac{\sigma^{2}}{2}\left(e^{2 T}-1\right)<\infty$ for any $T>0$. Moreover, from (1.4), it follows that for any $T>0$

$$
\int_{0}^{T} \int_{\Gamma} e^{2 s}[\ln (1+\gamma(z))]^{2} \lambda(\mathrm{d} z) \mathrm{d} s \leq K \int_{0}^{T} e^{2 s} \mathrm{~d} s=\frac{K}{2}\left(e^{2 T}-1\right)<\infty .
$$


By virtue of Lemma 3.1, for any $\vartheta, \varrho, T>0$, we have

$$
\begin{aligned}
\mathbb{P}\left\{\operatorname { s u p } _ { 0 \leq t \leq T } \left[\int_{0}^{t}-\sigma e^{s} \mathrm{~d} B(s)-\frac{\vartheta}{2} \int_{0}^{t} \sigma^{2} e^{2 s} \mathrm{~d} s+\int_{0}^{t} \int_{\Gamma} e^{s} \ln (1+\gamma(z)) \tilde{N}(\mathrm{~d} s, \mathrm{~d} z)\right.\right. \\
\left.\left.-\frac{1}{\vartheta} \int_{0}^{t} \int_{\Gamma}\left[(1+\gamma(z))^{\vartheta e^{s}}-1-\vartheta e^{s} \ln (1+\gamma(z))\right] \lambda(\mathrm{d} z) \mathrm{d} s\right]>\varrho\right\} \leq e^{-\vartheta \varrho} .
\end{aligned}
$$

Choose $T=n \gamma, \vartheta=\varepsilon e^{-n \gamma}$ and $\varrho=\left(\theta e^{n \gamma} \ln n\right) / \varepsilon$, where $n \in \mathbb{N}, \gamma>0, \theta>1$ and $0<\varepsilon<1$ in above equation. Since $\sum_{n=0}^{\infty} \frac{1}{n^{\theta}}<\infty$ for $\theta>1$, the Borel-Cantelli lemma (see Lem. 1.2.4 in [19]) implies that there exists a set $\Omega_{0} \in \mathcal{F}$ with $\mathbb{P}\left(\Omega_{0}\right)=1$ and an integer-valued random variable $n_{0}=n_{0}(\omega)$ such that for every $\omega \in \Omega_{0}$

$$
\begin{aligned}
\int_{0}^{t}-\sigma e^{s} \mathrm{~d} B(s)+\int_{0}^{t} \int_{\Gamma} e^{s} \ln (1+\gamma(z)) & \tilde{N}(\mathrm{~d} s, \mathrm{~d} z) \leq \frac{\theta e^{n \gamma} \ln n}{\varepsilon}+\frac{\varepsilon e^{-n \gamma}}{2} \int_{0}^{t} \sigma^{2} e^{2 s} \mathrm{~d} s \\
& +\frac{e^{n \gamma}}{\varepsilon} \int_{0}^{t} \int_{\Gamma}\left[(1+\gamma(z))^{\varepsilon e^{s-n \gamma}}-1-\varepsilon e^{s-n \gamma} \ln (1+\gamma(z))\right] \lambda(\mathrm{d} z) \mathrm{d} s
\end{aligned}
$$

holds for all $0 \leq t \leq n \gamma, n \geq n_{0}$. Substituting the above inequality into (3.1), we see that

$$
\begin{aligned}
e^{t} \ln x(t) \leq & \int_{0}^{t} e^{s}[\ln x(s)+r-c x(s)] \mathrm{d} s+\frac{\theta e^{n \gamma} \ln n}{\varepsilon}-\frac{1}{2} \int_{0}^{t} \sigma^{2} e^{s} \mathrm{~d} s+\frac{\varepsilon e^{-n \gamma}}{2} \int_{0}^{t} \sigma^{2} e^{2 s} \mathrm{~d} s \\
& +\frac{e^{n \gamma}}{\varepsilon} \int_{0}^{t} \int_{\Gamma}\left[(1+\gamma(z))^{\varepsilon e^{s-n \gamma}}-1-\varepsilon e^{s-n \gamma} \ln (1+\gamma(z))\right] \lambda(\mathrm{d} z) \mathrm{d} s+\ln x_{0}
\end{aligned}
$$

holds for all $0 \leq t \leq n \gamma, n \geq n_{0}$. For $0 \leq s \leq t \leq n \gamma$, we have $\frac{1}{2} \varepsilon e^{-n \gamma} \sigma^{2} e^{2 s}-\frac{1}{2} \sigma^{2} e^{s} \leq \frac{1}{2} \sigma^{2} e^{s}(\varepsilon-1)<0$ and

$$
\begin{aligned}
(1+\gamma(z))^{\varepsilon e^{s-n \gamma}}-1-\varepsilon e^{s-n \gamma} \ln (1+\gamma(z)) & \leq 1+\varepsilon e^{s-n \gamma} \gamma(z)-1-\varepsilon e^{s-n \gamma} \ln (1+\gamma(z)) \\
& =\varepsilon e^{s-n \gamma}[\gamma(z)-\ln (1+\gamma(z))]
\end{aligned}
$$

where in the second inequality, we use the inequality $x^{r} \leq 1+r(x-1), x \geq 0,1 \geq r \geq 0$. Therefore, for all $0 \leq t \leq n \gamma, n \geq n_{0}$, it follows from (3.2) that

$$
e^{t} \ln x(t) \leq \ln x_{0}+\int_{0}^{t} e^{s}\left[\ln x(s)-c x(s)+r+\int_{\Gamma}[\gamma(z)-\ln (1+\gamma(z))] \lambda(\mathrm{d} z)\right] \mathrm{d} s+\frac{\theta e^{n \gamma} \ln n}{\varepsilon} .
$$

Let us consider function $q(x)=\ln x-c x+r+\int_{\Gamma}[\gamma(z)-\ln (1+\gamma(z))] \lambda(\mathrm{d} z)$ on $(0, \infty)$. It is easy to show that $q$ has maximum value for $x=\frac{1}{c}>0$ and maximum value of $q$ is $q_{\max }=\ln \frac{1}{c}-1+r+\int_{\Gamma}[\gamma(z)+\ln (1+\gamma(z))] \lambda(\mathrm{d} z)$. Denote $H_{1} \doteq\left(\ln \frac{1}{c}-1+r+\int_{\Gamma}[\gamma(z)+\ln (1+\gamma(z))] \lambda(\mathrm{d} z)\right) \vee 1$. Then $e^{t} \ln x(t) \leq \ln x_{0}+H_{1} e^{t}+\frac{\theta e^{n \gamma} \ln n}{\varepsilon}$ holds for all $0 \leq t \leq n \gamma, n \geq n_{0}$. Therefore, for all $0 \leq(n-1) \gamma \leq t \leq n \gamma, n \geq n_{0}$, we have

$$
\frac{\ln x(t)}{\ln t} \leq \frac{\ln x_{0}}{e^{t} \ln t}+\frac{H_{1}}{\ln t}+\frac{\theta e^{\gamma} \ln n}{\varepsilon \ln [(n-1) \gamma]} .
$$

Letting $n \rightarrow \infty$ (and so $t \rightarrow \infty$ ), we obtain $\lim \sup _{t \rightarrow \infty} \frac{\ln x(t)}{\ln t} \leq \frac{\theta e^{\gamma}}{\varepsilon}$. Moreover, letting $\theta \downarrow 1, \gamma \downarrow 0$ and $\varepsilon \uparrow 1$,

$$
\limsup _{t \rightarrow \infty} \frac{\ln x(t)}{\ln t} \leq 1 \text { a.s. }
$$


The proof is complete.

Now, we show that model (1.3) is stochastically ultimate bounded.

Definition 3.3 (see $[12,13]$ ). Model $(1.3)$ is called stochastically ultimate bounded, if for any $\varepsilon \in(0,1)$, there exist a positive constant $H_{2}=H_{2}(\varepsilon)$ such that the solution $x(t)$ of model (1.3) with any initial value $x_{0} \in \mathbb{R}_{+}$ has the property that

$$
\limsup _{t \rightarrow \infty} \mathbb{P}\left\{x(t)>H_{2}\right\}<\varepsilon
$$

Theorem 3.4. For any $x_{0} \in \mathbb{R}_{+}$, let $x(t)$ be the solution of model (1.3) with initial value $x_{0}$. Then, for any $p \geq 1$, there is a constant $K(p)>0$ such that

$$
\mathbb{E}\left[x^{p}(t)\right] \leq K(p) .
$$

Further, model (1.3) is stochastically ultimate bounded.

Proof. For $p>1$, applying Itô's formula to $e^{t} x^{p}$ and taking expectations, we obtain

$$
\begin{aligned}
\mathbb{E}\left[e^{t} x^{p}(t)\right] & =x_{0}^{p}+p \mathbb{E} \int_{0}^{t} e^{s} x^{p}\left[\frac{1}{p}+r+\frac{p-1}{2} \sigma^{2}+\frac{1}{p} \int_{\Gamma}\left[(1+\gamma(z))^{p}-1-p \gamma(z)\right] \lambda(\mathrm{d} z)-c x(s)-\frac{a}{1+h a x(s)}\right] \mathrm{d} s \\
& \leq x_{0}^{p}+p \mathbb{E} \int_{0}^{t} e^{s} x^{p}(s)\left[\frac{1}{p}+r+\frac{p-1}{2} \sigma^{2}+\frac{1}{p} \int_{\Gamma}\left[(1+\gamma(z))^{p}-1-p \gamma(z)\right] \lambda(\mathrm{d} z)-c x(s)\right] \mathrm{d} s
\end{aligned}
$$

Denote $\kappa=\frac{1}{p}+r+\frac{p-1}{2} \sigma^{2}+\frac{1}{p} \int_{\Gamma}\left[(1+\gamma(z))^{p}-1-p \gamma(z)\right] \lambda(\mathrm{d} z)$. For $p>1$, we consider the function $f(s)=$ $(1+s)^{p}-1-p s$ on $(-1, \infty)$. Note that $f$ reaches its minimum value at $s=0$. Thus, we have $f(s) \geq 0$. This means that $\kappa>0$ for $p>1$. Clearly, if $p=1$, then $\kappa=1+r>0$. Therefore, for $p \geq 1$, we have

$$
\mathbb{E}\left[e^{t} x^{p}(t)\right] \leq x_{0}^{p}+p \int_{0}^{t} e^{s} \mathbb{E}\left\{x^{p}(s)[\kappa-c x(s)]\right\} \mathrm{d} s
$$

where $\kappa>0$ is a constant. Note that $g(x)=x^{p}(\kappa-c x)$ reaches its maximum value at $x=\frac{p \kappa}{c(p+1)} \geq 0$. Thus, $g_{\max }=\left(\frac{p}{c}\right)^{p}\left(\frac{\kappa}{p+1}\right)^{p+1} \doteq \kappa^{*}$ and $\mathbb{E}\left[e^{t} x^{p}(t)\right] \leq x_{0}^{p}+p \kappa^{*} \int_{0}^{t} e^{s} \mathrm{~d} s=x_{0}^{p}+p \kappa^{*}\left(e^{t}-1\right)$, which implies

$$
\mathbb{E}\left[x^{p}(t)\right] \leq x_{0}^{p} e^{-t}-p \kappa^{*} e^{-t}+p \kappa^{*} \leq x_{0}^{p}+p \kappa^{*} \doteq K(p) .
$$

Further, there is a positive constant $K(1)$ such that $\limsup _{t \rightarrow \infty} \mathbb{E}[x(t)] \leq K(1)$. Now, for any $\varepsilon \in(0,1)$, let $H_{2}=\frac{K(1)}{\varepsilon}+1$. Then by Markov's inequality,

$$
\mathbb{P}\left\{x(t)>H_{2}\right\} \leq \frac{\mathbb{E}[x(t)]}{H_{2}} .
$$

Hence,

$$
\limsup _{t \rightarrow \infty} \mathbb{P}\left\{x(t)>H_{2}\right\} \leq \limsup _{t \rightarrow \infty} \frac{\mathbb{E}[x(t)]}{H_{2}}<\varepsilon .
$$

The proof is complete. 


\section{ExtinCtion AND PERsistenCE}

In this part, we will investigate how Allee effect and stochastic perturbation affect the population dynamics behavior. For simplicity, denote $\langle u(t)\rangle=\frac{1}{t} \int_{0}^{t} u(s) \mathrm{d} s$.

\subsection{Extinction}

Theorem 4.1. For any initial value $x_{0} \in \mathbb{R}_{+}$, let $x(t)$ be the solution of model (1.3) with $x(0)=x_{0}$. If one of the following two conditions holds:

(i) $r+\beta<a \leq \sqrt{\frac{c}{h}}$ (the attack rate $a$ is in the intermediate range);

(ii) $a>\sqrt{\frac{c}{h}}$ and $a>\frac{c}{h} \frac{1}{2 \sqrt{\frac{c}{h}}-r-\beta}>0$ (the attack rate $a$ is large),

then the population becomes extinct exponentially with probability one.

Proof. Applying the generalized Itô formula, we obtain

$$
\ln x(t)=\ln x_{0}+\int_{0}^{t}\left[r-c x(s)-\frac{a}{h a x(s)+1}+\beta\right] \mathrm{d} s+\sigma B(t)+M(t) .
$$

Using the strong law of large numbers (see [19]), we have

$$
\lim _{t \rightarrow \infty} \frac{B(t)}{t}=0 \text { a.s. }
$$

In addition, $M(t)$ is a local martingale with $M(0)=0$ and the quadratic variation of $M(t)$ is $\langle M\rangle(t) \doteq\langle M, M\rangle_{t}=$ $\int_{0}^{t} \int_{\Gamma}[\ln (1+\gamma(z))]^{2} \lambda(\mathrm{d} z) \mathrm{d} s$. It follows from (1.4) that

$$
\rho_{M}(t) \doteq \int_{0}^{t} \frac{\mathrm{d}\langle M\rangle(s)}{(1+s)^{2}}=\int_{0}^{t} \frac{\int_{\Gamma}[\ln (1+\gamma(z))]^{2} \lambda(\mathrm{d} z)}{(1+s)^{2}} \mathrm{~d} s \leq \frac{K t}{1+t} .
$$

Thus, $\lim _{t \rightarrow \infty} \rho_{M}(t) \leq K<\infty$. From the strong law of large numbers for local martingales (see [15]),

$$
\lim _{t \rightarrow \infty} \frac{M(t)}{t}=0 \text { a.s. }
$$

Therefore, from (4.2) and (4.3), it follows that

$$
\lim _{t \rightarrow \infty} \frac{1}{t}\left[\ln x_{0}+\sigma B(t)+M(t)\right]=0 \text { a.s. }
$$

Consider $f(x)=r+\beta-c x-\frac{a}{h a x+1}$ on $[0,+\infty)$. Obviously, $f^{\prime}(x)=\frac{g(x)}{(\operatorname{hax}+1)^{2}}$, where $g(x)=-\operatorname{ch}^{2} a^{2} x^{2}-2 \operatorname{chax}+$ $h a^{2}-c$. By a simple calculation, we can obtain solutions of quadratic equation $g(x)=0$ are

$$
x_{1}=\frac{c+\sqrt{\operatorname{ch} a^{2}}}{-\operatorname{ch} a}, \quad x_{2}=\frac{c-\sqrt{\operatorname{ch} a^{2}}}{-\operatorname{ch} a} .
$$

Now, we differ two cases:

Case 1. (i) holds. From $a \leq \sqrt{\frac{c}{h}}$, it follows that $x_{1}<0$ and $x_{2} \leq 0$. Then, $g(x)<0$ for each $x \in(0,+\infty)$. This implies $f^{\prime}(x)<0$ on $(0, \infty)$. Thus, $f(x)<f(0)=r+\beta-a$ for all $x \in(0,+\infty)$. Now, from (4.1), it follows that

$$
\frac{1}{t} \ln x(t) \leq(r+\beta-a)+\frac{1}{t}\left[\ln x_{0}+\sigma B(t)+M(t)\right],
$$


which, together with (4.4), yields

$$
\limsup _{t \rightarrow \infty}\left[\frac{1}{t} \ln x(t)\right] \leq r+\beta-a<0 \text { a.s. }
$$

Case 2. (ii) holds. If $a>\sqrt{\frac{c}{h}}$, then $x_{1}<0$ and $x_{2}>0$. Thus, function $f(x)$ has maximum value at $x=x_{2}$, and $f_{\max }=r+\beta+\frac{c}{h a}-2 \sqrt{\frac{c}{h}}$. Thus, from (4.1), it follows that

$$
\frac{1}{t} \ln x(t) \leq\left(r+\beta+\frac{c}{h a}-2 \sqrt{\frac{c}{h}}\right)+\frac{1}{t}\left[\ln x_{0}+\sigma B(t)+M(t)\right]
$$

which, together with (4.4), yields

$$
\limsup _{t \rightarrow \infty}\left[\frac{1}{t} \ln x(t)\right] \leq r+\beta+\frac{c}{h a}-2 \sqrt{\frac{c}{h}} \text { a.s. }
$$

From $a>\frac{c}{h} \frac{1}{2 \sqrt{\frac{c}{h}}-r-\beta}>0$, we know $r+\beta+\frac{c}{h a}-2 \sqrt{\frac{c}{h}}<0$. The proof is complete.

From Theorem 4.1, one can get the following corollary.

Corollary 4.2. For any $x_{0} \in \mathbb{R}_{+}$, let $x(t)$ be the solution of model (1.3) corresponding to $x(0)=x_{0}$. If $r+\beta<0$, then $\lim _{t \rightarrow \infty} x(t)=0$. This means that the high intensity noise and jump can lead to the extinction of the population.

Proof. From $r+\beta<0$, it follows that $\frac{c}{h} \frac{1}{2 \sqrt{\frac{c}{h}}-r-\beta} \leq \frac{c}{h} \frac{1}{2 \sqrt{\frac{c}{h}}}=\frac{1}{2} \sqrt{\frac{c}{h}}<\sqrt{\frac{c}{h}}$. Thus, the condition (ii) in Theorem 4.1 equals to $a>\sqrt{\frac{c}{h}}$. Note that $r+\beta<0<a$. This, together with the condition (i) in Theorem 4.1, yields that if $r+\beta<0$, then $\lim \sup _{t \rightarrow \infty} \frac{\ln x(t)}{t}<0$. Hence, one can get the conclusion.

Moreover, for models (1.1) and (1.2), from the proof of Theorem 4.1, one can get the following two corollaries.

Corollary 4.3. For any $x_{0} \in \mathbb{R}_{+}$, let $x(t)$ be the solution of model (1.1) corresponding to $x(0)=x_{0}$. If one of the following conditions holds:

$\left(A_{1}\right) r<a \leq \sqrt{\frac{c}{h}}$

$\left(A_{2}\right) a>\sqrt{\frac{c}{h}}$ and $a>\frac{c}{h} \frac{1}{2 \sqrt{\frac{c}{h}-r}}>0$

then $\lim \sup _{t \rightarrow \infty} \frac{\ln x(t)}{t}<0$. That is, $\lim _{t \rightarrow \infty} x(t)=0$.

Corollary 4.4. For any $x_{0} \in \mathbb{R}_{+}$, let $x(t)$ be the solution of model (1.2) corresponding to $x(0)=x_{0}$. If one of the following conditions holds:

$\left(B_{1}\right) r-\frac{\sigma^{2}}{2}<a \leq \sqrt{\frac{c}{h}}$

$\left(B_{2}\right) a>\sqrt{\frac{c}{h}}$ and $a>\frac{c}{h} \frac{1}{2 \sqrt{\frac{c}{h}}-r+\frac{\sigma^{2}}{2}}>0$,

then $\lim \sup _{t \rightarrow \infty} \frac{\ln x(t)}{t}<0$ a.s. That is, $\lim _{t \rightarrow \infty} x(t)=0$ a.s.

Remark 4.5. Kang and Udiani [10] investigated the following single species model with Allee effects

$$
x^{\prime}(t)=x(t)\left[r\left(1-\frac{x(t)}{K}\right)-\frac{a}{1+h a x(t)}\right]
$$

with $x(0)=x_{0}$. Let $x^{K}=\frac{(h a K-1)+\sqrt{(h a K+1)^{2}-\frac{4 a^{2} h K}{r}}}{2 h a}$ and $x^{\theta}=\frac{(h a K-1)-\sqrt{(h a K+1)^{2}-\frac{4 a^{2} h K}{r}}}{2 h a}$. For model $(4.5)$, from Lemma 2.1 in [10], we know that 
(1) If $a<r$, then model (4.5) has the following two equilibria: $x^{0}=0$ and $x^{K}$, where $x^{0}=0$ is unstable while $x^{K}$ is locally asymptotically stable.

(2) If $r<a<\frac{1}{h K}$ or $\max \left\{r, \frac{1}{h K}, \sqrt{\frac{r}{h K}}\left(\frac{2+\sqrt{r h K}}{4-r h K}\right)\right\}<a$ and $r h K<4$, then model (4.5) has only the extinction equilibrium $x^{0}=0$ which is globally stable.

(3) If $4 h K<r<a$ hold or $\max \left\{r, \frac{1}{h K}\right\}<a<\sqrt{\frac{r}{h K}}\left(\frac{2+\sqrt{r h K}}{4-r h K}\right)$ and $r<\frac{4}{h K}$ hold, then (4.5) has three equilibria: $x^{0}, x^{K}$ and $x^{\theta}$, where both $x^{0}$ and $x^{K}$ are locally asymptotically stable while $x^{\theta}$ is unstable.

If we take $c=\frac{r}{K}$, then model (4.5) can be reduced to model (1.1). From Lemma 2.1 (2) in [10], for any initial value $x_{0} \in \mathbb{R}_{+}$, if one of the following conditions holds:

$\left(A_{3}\right) r<a<\frac{c}{r h}$

$\left(A_{4}\right) 4 c>r^{2} h$ and $\max \left\{r, \frac{c}{r h}, \sqrt{\frac{c}{h}} \frac{1}{2 \sqrt{\frac{c}{h}-r}}\right\}<a$

then the solution $x(t)$ of model (1.1) corresponding to $x(0)=x_{0}$ satisfies $\lim _{t \rightarrow \infty} x(t)=0$.

Obviously, if condition $\left(A_{3}\right)$ or $\left(A_{4}\right)$ holds, then condition $\left(A_{1}\right)$ or $\left(A_{2}\right)$ in Corollary 4.3 holds. Furthermore, from $\left(A_{3}\right)$ and $\left(A_{4}\right)$, it follows that $a \neq \frac{c}{r h}$. However, if $a=\frac{c}{r h}$ and $a>\sqrt{\frac{c}{h}}$, then $\frac{c}{r h}>\sqrt{\frac{c}{h}}$. This means that $r<\sqrt{\frac{c}{h}}$. Thus, we have $2 \sqrt{\frac{c}{h}}-r>r$. This implies that $0<\frac{c}{h} \frac{1}{2 \sqrt{\frac{c}{h}}-r}<\frac{c}{r h}=a$. Thus, condition $\left(A_{2}\right)$ holds. Therefore, Corollary 4.3 generalizes and improves Lemma 2.1 in [10].

Remark 4.6. In [26], the authors discussed the persistence and ergodicity of the following stochastic model under regime switching

$$
\mathrm{d} x(t)=x(t)\left[r(\xi(t))\left(1-\frac{x(t)}{K(\xi(t))}\right)-\frac{a(\xi(t))}{1+h(\xi(t)) a(\xi(t)) x(t)}\right] \mathrm{d} t+\sigma(\xi(t)) \mathrm{d} B(t),
$$

where $r(i), K(i), a(i)$ and $h(i)$ are all positive for any $i \in \mathcal{M}=\{1,2, \cdots, N\}$, and $\xi(t)$ is $\mathcal{F}_{t}$ adapted but independent of the Brownian motion $B(t)$. Let $b(\xi(t))=r(\xi(t))-\frac{1}{2} \sigma^{2}(\xi(t)), \bar{a}=\sum_{i=1}^{N} \pi_{i} a(i), \bar{b}=\sum_{i=1}^{N} \pi_{i} b(i)$ and $\bar{r}=\sum_{i=1}^{N} \pi_{i} \sqrt{\frac{r(i)}{h(i) K(i)}}$. Then, for model (4.6), from Theorem 3.1 in [26], we know that

(a) If $\min _{i \in \mathcal{M}}\left[\frac{r(i)}{K(i)}-h(i) a^{2}(i)\right]>0$, we have

(a1) $\lim _{t \rightarrow \infty} x(t)=0$, i.e., the population is extinct when $\bar{b}<\bar{a}$;

(a2) $\lim _{t \rightarrow \infty} \frac{1}{t} \int_{0}^{t} x(s) \mathrm{d} s=0$, i.e., the population is nonpersistent in mean when $\bar{b}=\bar{a}$;

(a3) $\liminf _{t \rightarrow \infty} \frac{1}{t} \int_{0}^{t} x(s) \mathrm{d} s>0$, i.e., the population is persistent in mean when $\bar{b}>\bar{a}$.

(b) If $\min _{i \in \mathcal{M}}\left[\frac{r(i)}{K(i)}-h(i) a^{2}(i)\right] \leq 0$, we have

(b1) $\lim _{t \rightarrow \infty} x(t)=0$, i.e., the population is extinct when $\bar{b}<\bar{r}$;

(b2) $\liminf _{t \rightarrow \infty} \frac{1}{t} \int_{0}^{t} x(s) \mathrm{d} s>0$, i.e., the population is persistent in mean when $\bar{b}>\bar{a}$.

If there is only one state in the state space $\mathcal{M}$ and $c=\frac{r}{K}$, then model (4.6) can be reduced to model (1.2).

From Theorem 3.1 in [26], for any initial value $x_{0} \in \mathbb{R}_{+}$, if one of the following conditions holds:

$\left(B_{3}\right) r-\frac{\sigma^{2}}{2}<a \leq \sqrt{\frac{c}{h}}$;

$\left(B_{4}\right) a>\sqrt{\frac{c}{h}}$ and $r-\frac{\sigma^{2}}{2}<\sqrt{\frac{c}{h}}$

then the solution $x(t)$ of model (1.2) with $x(0)=x_{0}$ satisfies $\lim _{t \rightarrow \infty} x(t)=0$ a.s.

Obviously, if condition $\left(B_{3}\right)$ or $\left(B_{4}\right)$ holds, then condition $\left(B_{1}\right)$ or $\left(B_{2}\right)$ in Corollary 4.4 holds. However, from $\left(B_{2}\right)$ of Corollary 4.4, it follows that $r-\frac{\sigma^{2}}{2}<2 \sqrt{\frac{c}{h}}-\frac{c}{h a}$ and $a>\sqrt{\frac{c}{h}}$. Note that $2 \sqrt{\frac{c}{h}}-\frac{c}{h a}>\sqrt{\frac{c}{h}}$ when $a>\sqrt{\frac{c}{h}}$. Therefore, if there is only one state in the state space $\mathcal{M}$ and $c=\frac{r}{K}$ for the model in [26], then Corollary 4.4 is more general than Theorem 3.1 in [26]. 
Remark 4.7. Note that $\beta \leq-\frac{\sigma^{2}}{2}$. Thus, under the condition of Corollary 4.3, the solutions of models (1.1), (1.2) and (1.3) all tend to 0 exponentially. Meanwhile, under the condition of Corollary 4.4, the solutions of models (1.2) and (1.3) both tend to 0 exponentially. Moreover, from Corollary 4.3, it follows that no matter how loud or small the noise, if the attack rate $a$ is in the intermediate range or very large, i.e. $r<a \leq \sqrt{\frac{c}{h}}$ or $a>\sqrt{\frac{c}{h}}$ and $a>\frac{c}{h} \frac{1}{2 \sqrt{\frac{c}{h}}-r}$, then the populations in models (1.1), (1.2) and (1.3) will go extinct (see Figs. 1 and 2). Comparison Theorem 4.1, Corollary 4.3 and Corollary 4.4 show that if attack rate $a$ is less than growth rate $r$ and the noise intensity or jump is relatively large, the populations in models (1.2) and (1.3) will become extinct (see Fig. 3).

\subsection{Persistence}

Lemma 4.8 (see $[26]$ ). Assume $u(t) \in C\left(\Omega \times \overline{\mathbb{R}}_{+}, \mathbb{R}_{+}\right), G(t) \in C\left(\Omega \times \overline{\mathbb{R}}_{+},(-\infty,+\infty)\right)$, $\lim _{t \rightarrow \infty} \frac{G(t)}{t}=0$ a.s.

(I) If there are $\varrho>0, \varrho_{0}>0$ and $T>0$ satisfying

$$
\ln u(t) \leq \varrho t-\varrho_{0} \int_{0}^{t} u(s) \mathrm{d} s+G(t), \text { a.s., } t \geq T,
$$

then $\lim \sup _{t \rightarrow \infty}\langle u(t)\rangle \leq \frac{\varrho}{\varrho_{0}}$ a.s.

(II) If there exist $\varrho>0, \varrho_{0}>0$ and $T>0$ satisfying

$$
\ln u(t) \geq \varrho t-\varrho_{0} \int_{0}^{t} u(s) \mathrm{d} s+G(t), \text { a.s., } t \geq T,
$$

then $\liminf \operatorname{in}_{t \rightarrow \infty}\langle u(t)\rangle \geq \frac{\varrho}{\varrho_{0}}$ a.s.

Theorem 4.9. For any $x_{0} \in \mathbb{R}_{+}$, let $x(t)$ be the solution of model (1.3) with $x(0)=x_{0}$. If $r+\beta-a>0$, then

$$
\limsup _{t \rightarrow \infty}\langle x(t)\rangle \leq \frac{r+\beta}{c}, \quad \liminf _{t \rightarrow \infty}\langle x(t)\rangle \geq \frac{r+\beta-a}{c} \text { a.s. }
$$

That is, population $x(t)$ in model (1.3) is persistent in mean.

Proof. It follows from (4.1) that

$$
\ln x(t)=\ln x_{0}+\int_{0}^{t}\left[r-c x(s)-\frac{a}{h a x(s)+1}+\beta\right] \mathrm{d} s+\sigma B(t)+M(t) .
$$

Thus, we have

$$
\begin{aligned}
& \ln x(t) \leq(r+\beta) t-c \int_{0}^{t} x(s) \mathrm{d} s+\ln x_{0}+\sigma B(t)+M(t), \\
& \ln x(t) \geq(r+\beta-a) t-c \int_{0}^{t} x(s) \mathrm{d} s+\ln x_{0}+\sigma B(t)+M(t) .
\end{aligned}
$$

From (4.4), $r+\beta-a>0$ and Lemma 4.8, we have

$$
\limsup _{t \rightarrow \infty}\langle x(t)\rangle \leq \frac{r+\beta}{c}, \quad \liminf _{t \rightarrow \infty}\langle x(t)\rangle \geq \frac{r+\beta-a}{c} \text { a.s. }
$$

The proof is complete. 
From Theorem 4.9, one can get the following result.

Corollary 4.10. For any $x_{0} \in \mathbb{R}_{+}$, let $x(t)$ be the solution of model (1.2) with $x(0)=x_{0}$. If $r-\frac{\sigma^{2}}{2}-a>0$, then

$$
\limsup _{t \rightarrow \infty}\langle x(t)\rangle \leq \frac{r-\frac{\sigma^{2}}{2}}{c}, \quad \liminf _{t \rightarrow \infty}\langle x(t)\rangle \geq \frac{r-\frac{\sigma^{2}}{2}-a}{c} \text { a.s. }
$$

Remark 4.11. From Theorem 4.9, if attack rate $a$ is less than growth rate $r$ and the noise intensity or jump is relatively small such that $a<r+\beta$, the population in model (1.3) will be persistent in mean (see Fig. 4).

Remark 4.12. If there is no Allee effect, then stochastic model (1.3) can be reduced to

$$
\mathrm{d} x(t)=x(t-)[r-c x(t-)] \mathrm{d} t+\sigma x(t-) \mathrm{d} B(t)+\int_{\Gamma} x(t-) \gamma(z) \tilde{N}(\mathrm{~d} t, \mathrm{~d} z) .
$$

For stochastic model (4.7), from Theorems 4.1 and 4.9, one can get the following results

(i) if $r+\beta<0$, then $\lim _{t \rightarrow \infty} x(t)=0$ a.s.;

(ii) if $r+\beta>0$, then $\lim _{t \rightarrow \infty}\langle x(t)\rangle=\frac{r+\beta}{c}$ a.s.

$\mathrm{Wu}$ and Wang [24] discussed non-autonomous model corresponding to model (4.7). From [24], we know that the jump noise and the general noise can make the population extinct. [31] discussed the dynamics of a stochastic predator-prey model with habitat complexity and prey aggregation. The results show that environmental noise is disadvantage for the survival of biological population.

\section{Global attractivity AND Stability IN Distribution}

In this section, we first show that the solution of model (1.3) is globally attractive in mean. Then we consider the stability in distribution of model (1.3).

Definition 5.1. For any $x_{i 0} \in \mathbb{R}_{+}$, let $x_{i}(t)$ be the solution of model $(1.3)$ with $x_{i}(0)=x_{i 0}(i=1,2)$. If $\lim _{t \rightarrow \infty} \mathbb{E}\left|x_{1}(t)-x_{2}(t)\right|=0$, then the solution of model (1.3) is globally attractive in mean.

Let $x\left(t ; x_{0}\right)$ be the solution of model (1.3) corresponding to $x(0)=x_{0} \in \mathbb{R}_{+}$and $p\left(t, x_{0}, \cdot\right)$ be the transition probability of $x\left(t ; x_{0}\right)$. Then, for any $A \in \mathcal{B}\left(\mathbb{R}_{+}\right)$, we have

$$
P\left(t, x_{0}, A\right)=\mathbb{P}\left\{x\left(t ; x_{0}\right) \in A\right\}=\int_{A} p\left(t, x_{0}, \mathrm{~d} \eta\right) .
$$

Let $\mathcal{P}\left(\mathbb{R}_{+}\right)$be the family of probability measures on the measurable space $\left(\mathbb{R}_{+}, \mathcal{B}\left(\mathbb{R}_{+}\right)\right)$. For any $P_{1}, P_{2} \in \mathcal{P}\left(\mathbb{R}_{+}\right)$, define the metric

$$
\mathrm{d}_{\mathbb{S}}\left(P_{1}, P_{2}\right)=\sup _{f \in \mathbb{S}}\left|\int_{\mathbb{R}_{+}} f(s) P_{1}(\mathrm{~d} s)-\int_{\mathbb{R}_{+}} f(s) P_{2}(\mathrm{~d} s)\right|,
$$

where $\mathbb{S}=\left\{f: \mathbb{R}_{+} \rightarrow \mathbb{R}|| f\left(s_{1}\right)-f\left(s_{2}\right)|\leq| s_{1}-s_{2} \mid\right.$ and $|f(\cdot)| \leq 1$ for $\left.s_{1}, s_{2} \in \mathbb{R}_{+}\right\}$. Thus, $\left(\mathcal{P}\left(\mathbb{R}_{+}\right), \mathrm{d}_{\mathbb{S}}\right)$ is a complete metric space.

Definition 5.2 (see [23]). If there is $\nu(\cdot) \in \mathcal{P}\left(\mathbb{R}_{+}\right)$such that for each $x_{0} \in \mathbb{R}_{+}, p\left(t, x_{0}, \cdot\right)$ converges weakly to $\nu(\cdot)$ as $t \rightarrow \infty$, that is $\lim _{t \rightarrow \infty} \mathrm{d}_{\mathbb{S}}\left(p\left(t, x_{0}, \cdot\right), \nu(\cdot)\right)=0$, then model (1.3) is stable in distribution.

Lemma 5.3 (see [3]). If $f: \overline{\mathbb{R}}_{+} \rightarrow \overline{\mathbb{R}}_{+}$is uniformly continuous and integrable, then $\lim _{t \rightarrow \infty} f(t)=0$.

Lemma 5.4. For any $x_{i 0} \in \mathbb{R}_{+}$, let $x_{i}(t)$ be the solution of model $(1.3)$ with $x_{i}(0)=x_{i 0}(i=1,2)$. Then, $\mathbb{E}\left|x_{1}(t)-x_{2}(t)\right|$ is uniformly continuous on $[0, \infty)$. 
Proof. From (1.3), it follows that

$$
\mathbb{E}\left[x_{i}(t)\right]=x_{i 0}+\int_{0}^{t} r \mathbb{E}\left[x_{i}(s)\right]-c \mathbb{E}\left[x_{i}^{2}(s)\right]-\mathbb{E}\left[\frac{a x_{i}(s)}{h a x_{i}(s)+1}\right] \mathrm{d} s,
$$

which, together with Theorem 3.4, yields

$$
\begin{aligned}
& \frac{\mathrm{d} \mathbb{E}\left[x_{1}(t)\right]}{\mathrm{d} t}=r \mathbb{E}\left[x_{1}(t)\right]-c \mathbb{E}\left[x_{1}^{2}(t)\right]-\mathbb{E}\left[\frac{a x_{1}(t)}{h a x_{1}(t)+1}\right] \leq r \mathbb{E}\left[x_{1}(t)\right] \leq r K(1), \\
& \frac{\mathrm{d} \mathbb{E}\left[x_{1}(t)\right]}{\mathrm{d} t}=r \mathbb{E}\left[x_{1}(t)\right]-c \mathbb{E}\left[x_{1}^{2}(t)\right]-\mathbb{E}\left[\frac{a x_{1}(t)}{h a x_{1}(t)+1}\right] \geq-c \mathbb{E}\left[x_{1}^{2}(t)\right]-a \geq-c K(2)-a .
\end{aligned}
$$

Thus, we have $\left|\frac{\mathrm{d} \mathbb{E}\left[x_{1}(t)\right]}{\mathrm{d} t}\right| \leq M$, where $M=\max \{|r K(1)|,|-c K(2)-a|\}$. This implies that $\mathbb{E}\left[x_{1}(t)\right]$ is uniformly continuous. Similarly, $\mathbb{E}\left[x_{2}(t)\right]$ is also uniformly continuous. Therefore, for arbitrary $\varepsilon>0$, there is $\delta=\delta(\varepsilon)>0$ such that for each $0 \leq s, t<\infty$ with $|t-s|<\delta$,

$$
\left|\mathbb{E}\left[x_{i}(t)\right]-\mathbb{E}\left[x_{i}(s)\right]\right|<\frac{\varepsilon}{2}, \quad i=1,2 .
$$

Now, we show that $\mathbb{E}\left|x_{1}(t)-x_{2}(t)\right|$ is uniformly continuous. Without a loss of generality, set $x_{10} \geq x_{20}$. From the stochastic comparison theorem ([20], Thm. 3.1), it follows that $x_{1}(t) \geq x_{2}(t)$ a.s. for $t \in[0, \infty)$. Therefore,

$$
\mathbb{E}\left|x_{1}(t)-x_{2}(t)\right|=\mathbb{E}\left[x_{1}(t)-x_{2}(t)\right] .
$$

Furthermore, for each $0 \leq s, t<\infty$ with $|t-s|<\delta$, we have

$$
\begin{aligned}
|\mathbb{E}| x_{1}(t)-x_{2}(t)|-\mathbb{E}| x_{1}(s)-x_{2}(s)|| & =\left|\mathbb{E}\left[x_{1}(t)-x_{2}(t)\right]-\mathbb{E}\left[x_{1}(s)-x_{2}(s)\right]\right| \\
& =\left|\mathbb{E}\left[x_{1}(t)-x_{1}(s)\right]-\mathbb{E}\left[x_{2}(t)-x_{2}(s)\right]\right| \\
& \leq\left|\mathbb{E} x_{1}(t)-\mathbb{E} x_{1}(s)\right|+\left|\mathbb{E} x_{2}(t)-\mathbb{E} x_{2}(s)\right| \\
& <\varepsilon
\end{aligned}
$$

which implies the conclusion of the lemma.

Theorem 5.5. If $a<\sqrt{\frac{c}{h}}$, then the solution of model (1.3) is globally attractive in mean.

Proof. For any $x_{i 0} \in \mathbb{R}_{+}$, let $x_{i}(t)$ be the solution of model (1.3) corresponding to $x_{i}(0)=x_{i 0}(i=1,2)$. From the generalized Itô formula, it follows that

$$
\mathrm{d} \ln x_{i}(t)=\left[r-c x_{i}(t)-\frac{a}{1+h a x_{i}(t)}+\beta\right] \mathrm{d} t+\sigma \mathrm{d} B(t)+\int_{\Gamma} \ln (1+\gamma(z)) \tilde{N}(\mathrm{~d} t, \mathrm{~d} z), \quad i=1,2 .
$$

Set $V_{2}(t)=\left|\ln x_{1}(t)-\ln x_{2}(t)\right|$. Thus,

$$
\mathrm{d}^{+} V_{2}(t)=-c\left|x_{1}(t)-x_{2}(t)\right| \mathrm{d} t+\frac{h a^{2}\left|x_{1}(t)-x_{2}(t)\right|}{\left(1+h a x_{1}(t)\right)\left(1+h a x_{2}(t)\right)} \mathrm{d} t .
$$

Consequently,

$$
0 \leq \mathbb{E} V_{2}(t) \leq V_{2}(0)-\left(c-h a^{2}\right) \int_{0}^{t} \mathbb{E}\left|x_{1}(s)-x_{2}(s)\right| \mathrm{d} s
$$


That is $\left(c-h a^{2}\right) \int_{0}^{t} \mathbb{E}\left|x_{1}(s)-x_{2}(s)\right| \mathrm{d} s \leq V_{2}(0)<\infty$. It follows from $a<\sqrt{\frac{c}{h}}$ that $\mathbb{E}\left|x_{1}(t)-x_{2}(t)\right|$ is integrable on $[0, \infty)$. From Definition 5.1 and Lemmas 5.3 and 5.4, we know that Theorem 5.5 holds.

Corollary 5.6. If $a<\sqrt{\frac{c}{h}}$, then the solution of model (1.2) is globally attractive in mean.

Remark 5.7. From Theorem 5.5 and Corollary 5.6, if attack rate $a$ relatively small, i.e., $a<\sqrt{\frac{c}{h}}$, then the solution of model (1.2) is globally attractive in mean. For model (1.3), one can obtain the same result (see Fig. 5).

Theorem 5.8. If $a<\sqrt{\frac{c}{h}}$, then model (1.3) is stable in distribution.

Proof. For arbitrary $x_{0} \in \mathbb{R}_{+}, f \in \mathbb{S}$ and $t, s>0$, we have

$$
\left|\mathbb{E} f\left(x\left(t+s ; x_{0}\right)\right)-\mathbb{E} f\left(x\left(t ; x_{0}\right)\right)\right| \leq \int_{B_{\theta}}\left|\mathbb{E} f\left(x\left(t ; x_{0}\right)\right)-\mathbb{E} f\left(x\left(t ; y_{0}\right)\right)\right| p\left(s, x_{0}, \mathrm{~d} y_{0}\right)+2 P\left(s, x_{0}, \bar{B}_{\theta}\right),
$$

where $\theta>0, x_{0} \in B_{\theta}=\left[\frac{1}{\theta}, \theta\right]$ and $\bar{B}_{\theta}=\left(0, \frac{1}{\theta}\right) \cup(\theta, \infty)$. According to Chebyshev's inequality and Theorem 3.4, $\left\{p\left(t, x_{0}, \mathrm{~d} \eta\right): t \geq 0\right\}$ is tight. Thus, there is a sufficiently large $\theta$ such that

$$
P\left(s, x_{0}, \bar{B}_{\theta}\right) \leq \frac{\varepsilon}{4}, \quad s>0
$$

From Theorem 5.5, for any $y_{0} \in B_{\theta}$, there is $T>0$ such that $\mathbb{E}\left|x\left(t ; x_{0}\right)-x\left(t ; y_{0}\right)\right| \leq \frac{\varepsilon}{2}$ for $t>T$. Thus, for any $f \in \mathbb{S}$ and $t>T$, from the inequality $|\mathbb{E} x| \leq \mathbb{E}|x|$, it follows that

$$
\begin{aligned}
\int_{B_{\theta}}\left|\mathbb{E} f\left(x\left(t ; x_{0}\right)\right)-\mathbb{E} f\left(x\left(t ; y_{0}\right)\right)\right| p\left(s, x_{0}, \mathrm{~d} y_{0}\right) & \leq \int_{B_{\theta}} \mathbb{E}\left|f\left(x\left(t ; x_{0}\right)\right)-f\left(x\left(t ; y_{0}\right)\right)\right| p\left(s, x_{0}, \mathrm{~d} y_{0}\right) \\
& \leq \int_{B_{\theta}} \mathbb{E}\left|x\left(t ; x_{0}\right)-x\left(t ; y_{0}\right)\right| p\left(s, x_{0}, \mathrm{~d} y_{0}\right) \\
& \leq \frac{\varepsilon}{2} \int_{B_{\theta}} p\left(s, x_{0}, \mathrm{~d} y_{0}\right) \leq \frac{\varepsilon}{2} .
\end{aligned}
$$

From (5.1)-(5.3) and the arbitrariness of $f$, it follows that $\mathrm{d}_{\mathbb{S}}\left(p\left(t+s, x_{0}, \cdot\right), p\left(t, x_{0}, \cdot\right)\right) \leq \varepsilon$ for any $t>T, s>0$. Hence, for any $x_{0} \in \mathbb{R}_{+},\left\{p\left(t, x_{0}, \cdot\right): t \geq 0\right\}$ is a Cauchy sequence in $\left(\mathcal{P}\left(\mathbb{R}_{+}\right), \mathrm{d}_{\mathbb{S}}\right)$. Thus, there is a unique $\nu(\cdot) \in \mathcal{P}\left(\mathbb{R}_{+}\right)$, such that

$$
\lim _{t \rightarrow \infty} \mathrm{d}_{\mathbb{S}}(p(t, 0.01, \cdot), \nu(\cdot))=0
$$

For any $x_{0} \in \mathbb{R}_{+}$, a similar discussion, we have $\lim _{t \rightarrow \infty} \mathrm{d}_{\mathbb{S}}\left(p\left(t, x_{0}, \cdot\right), p(t, 0.01, \cdot)\right)=0$. This, together with (5.4) and triangle inequality, yields

$$
\limsup _{t \rightarrow \infty} \mathrm{d}_{\mathbb{S}}\left(p\left(t, x_{0}, \cdot\right), \nu(\cdot)\right) \leq \lim _{t \rightarrow \infty} \mathrm{d}_{\mathbb{S}}\left(p\left(t, x_{0}, \cdot\right), p(t, 0.01, \cdot)\right)+\lim _{t \rightarrow \infty} \mathrm{d}_{\mathbb{S}}(p(t, 0.01, \cdot), \nu(\cdot))=0 .
$$

Hence, $\lim _{t \rightarrow \infty} \mathrm{d}_{\mathbb{S}}\left(p\left(t, x_{0}, \cdot\right), \nu(\cdot)\right)=0$. By Definition 5.2 we complete the proof.

Corollary 5.9. If $a<\sqrt{\frac{c}{h}}$, then model (1.2) is stable in distribution.

Remark 5.10. From Theorem 5.8 and Corollary 5.9, if the attack rate $a$ relatively small, i.e., $a<\sqrt{\frac{c}{h}}$, then model (1.2) is stable in distribution. For model (1.3), one can get the same result (see Fig. 6). 


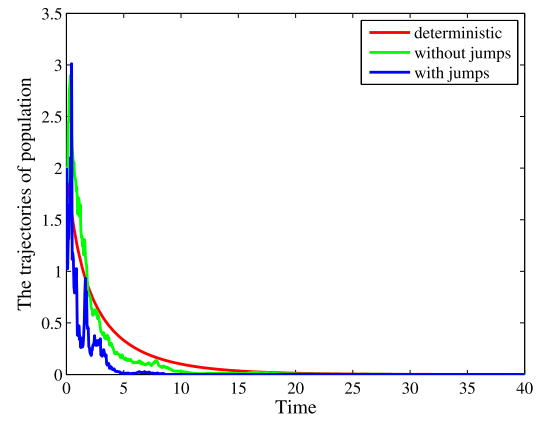

(a) $\sigma=0.3$ and $\gamma(z)=0.8$.

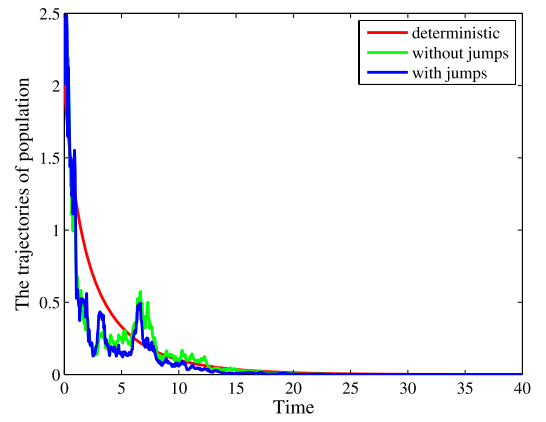

(b) $\sigma=0.5$ and $\gamma(z)=0.4$.

Figure 1. Deterministic and stochastic trajectories of the populations in models (1.1)-(1.3).

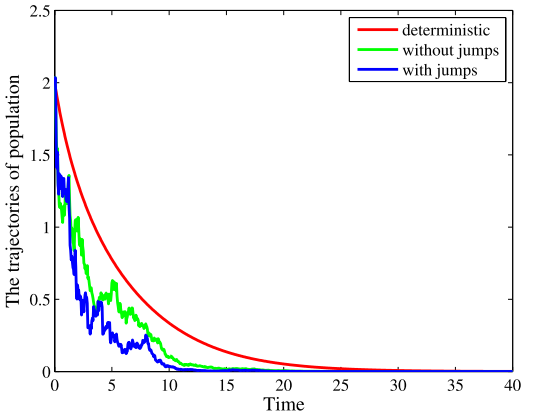

(a) $\sigma=0.3$ and $\gamma(z)=0.4$.

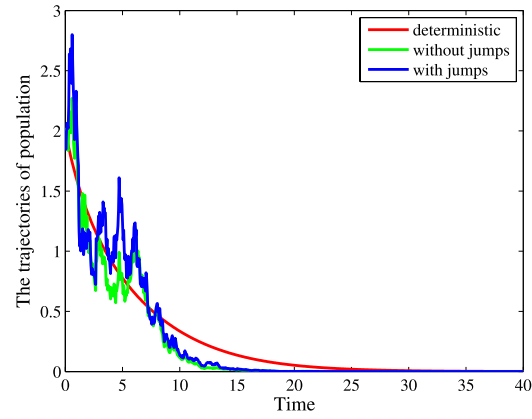

(b) $\sigma=0.4$ and $\gamma(z)=0.2$.

Figure 2. Deterministic and stochastic trajectories of the populations in models (1.1)-(1.3).

\section{NumERICAL SIMULATIONS}

In this part, using the theory and method (stationary Poisson point processes) mentioned in [33], we give some numerical simulations. Numerical experiments are made by using $\Gamma=(0,+\infty)$ and $\lambda(\Gamma)=1$. Denote $A=\frac{c}{h} \frac{1}{2 \sqrt{\frac{c}{h}}-r}, B=\frac{c}{h} \frac{1}{2 \sqrt{\frac{c}{h}-r+\frac{\sigma^{2}}{2}}}$ and $C=\frac{c}{h} \frac{1}{2 \sqrt{\frac{c}{h}-r-\beta}}$.

Example 6.1. From Theorem 4.1, it follows that no matter how large the noise intensity or jump is, as long as $r<a \leq \sqrt{\frac{c}{h}}$, or $a>\sqrt{\frac{c}{h}}$ and $a>A>0$, the population will be extinct with probability 1 . Here we choose $x_{0}=2, r=0.6, c=0.2, a=0.8$. In Figure 1, we choose $h=0.02$. By computing, we obtain $r<a \leq \sqrt{\frac{c}{h}}=\sqrt{10}$. In Figure 2, we choose $h=0.5$. By computing, we obtain $\sqrt{\frac{c}{h}}=\sqrt{0.4} \approx 0.6325<a$ and $0<A=\frac{0.4}{2 \sqrt{0.4}-0.6} \approx$ $0.602<a$. Therefore, from Theorem 4.1, the population will go to extinct.

From Figures 1 and 2, we can see that the solutions of models (1.1), (1.2) and (1.3) will tend to 0. This is consistent with Corollaries 4.3-4.4 and Theorem 4.1. The results show that if the attack rate $a$ is in the intermediate range or very large, then the populations in models (1.1), (1.2) and (1.3) will go extinct.

Example 6.2. Let $x_{0}=2, r=0.8$ and $c=0.1$. In Figure 3a, we choose $a=1, h=0.2, \sigma=0.2$ and $\gamma(z)=0.2$. By computing, $a>\sqrt{\frac{c}{h}} \approx 0.707$ and $a>A \approx 0.814>0$. Thus, by Theorem 4.1, populations in models (1.1)(1.3) are extinct with probability 1 . In Figure 3b, we choose $a=0.6, h=0.4, \sigma=1$ and $\gamma(z)=0.5$. Note that $a<r$. Then from Lemma 2.1 in [10], model (1.1) has equilibrium $x^{*}=5.38125$. Then $a>\sqrt{\frac{c}{h}} \approx 0.5$, $a<A=1.25$, and $a>B \approx 0.357$. Thus, by Theorem 4.1, the solutions of (1.2) and (1.3) will tend to 0 . In Figure 3c, set $a=0.51, h=0.4, \sigma=0.1$ and $\gamma(z)=1$. Note that $a<r$. Then from Lemma 2.1 in [10], 


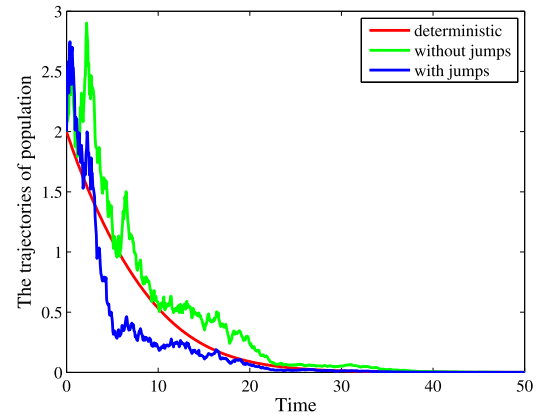

(a) $a=1, h=0.2, \sigma=0.2, \gamma(z)=0.2$.

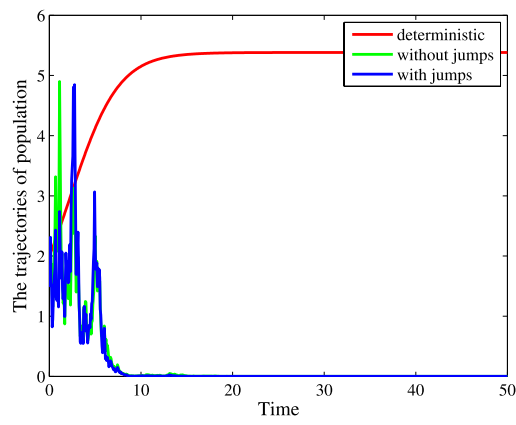

(b) $a=0.6, h=0.4, \sigma=1, \gamma(z)=0.5$.

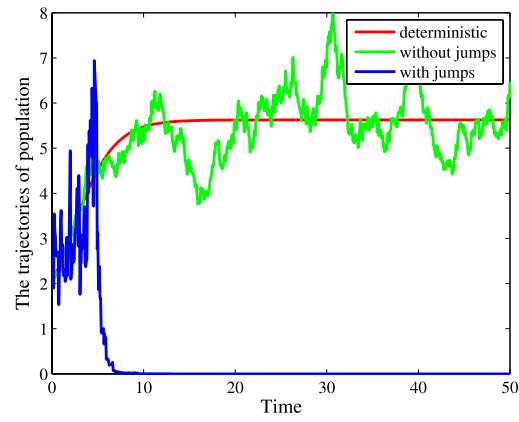

(c) $a=0.51, h=0.4, \sigma=0.1, \gamma(z)=1$

Figure 3. Deterministic and stochastic trajectories of the populations in models (1.1)-(1.3).

model (1.1) has equilibrium $x^{*}=5.625$. Then $\beta \approx-0.312, a>\sqrt{\frac{c}{h}}=0.5, a<A=1.25, a<B \approx 1.22$ and $a>C \approx 0.488$. Thus, by Theorem 4.1, the solution of (1.3) will tend to 0 .

Figure 3 shows that white noise and jump may affect the survival of the population. Moreover, the results show that if the attack rate $a$ is less than the growth rate $r$ and the noise intensity or jump is relatively large, the populations in models (1.2) and (1.3) will become extinct.

Example 6.3. In Figure 4, we choose $x_{0}=2, r=0.8, c=0.2, a=0.4, h=0.2, \sigma=0.2$ and $\gamma(z)=0.2$. Note that $a<r$. Then from Lemma 2.1 in [10], model (1.1) has equilibrium $x^{*}=2.312$. Thus, we have $\beta \approx-0.038$. Moreover, $r+\beta-a=0.8-0.038-0.4>0$. Then, by Theorem 4.9, the populations described by (1.1), (1.2) and (1.3) will be persistent in mean. Moreover, from Corollary 4.10 and Theorem 4.9, it follows that

$$
\begin{array}{r}
1.9 \leq \liminf _{t \rightarrow \infty}\langle x(t)\rangle \leq \limsup _{t \rightarrow \infty}\langle x(t)\rangle \leq 3.9 \text { for model }(1.2), \\
1.81 \leq \liminf _{t \rightarrow \infty}\langle x(t)\rangle \leq \limsup _{t \rightarrow \infty}\langle x(t)\rangle \leq 3.81 \text { for model }(1.3) .
\end{array}
$$

As can be seen from Figure 4a-c, populations described by models (1.1), (1.2) and (1.3) will be persistent in mean. The results show that if the attack rate $a$ is less than the average growth rate $r$ and the noise intensity or jump is relatively small, populations in models (1.1), (1.2) and (1.3) will be persistent in mean. Moreover, from Figure 4d, one can see that Allee effect would have an adverse effect on the survival of the population.

Example 6.4. In Figure 5, choose $r=0.8, c=0.12, a=0.5, h=0.4, \sigma=0.1$ and $\gamma(z)=0.2$. Thus, $0.1=$ $h a^{2}<c=0.12$. Then by Theorem 5.5, the solution of model (1.3) is globally attractive in mean. 


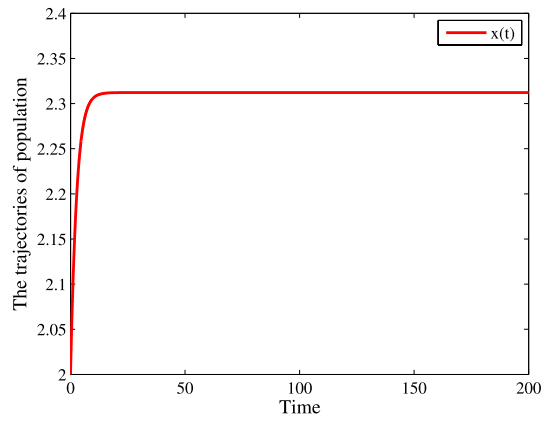

(a) Trajectories of model (1).

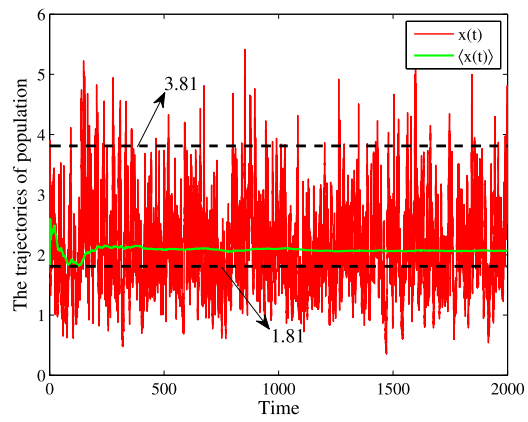

(c) Trajectories of model (3).

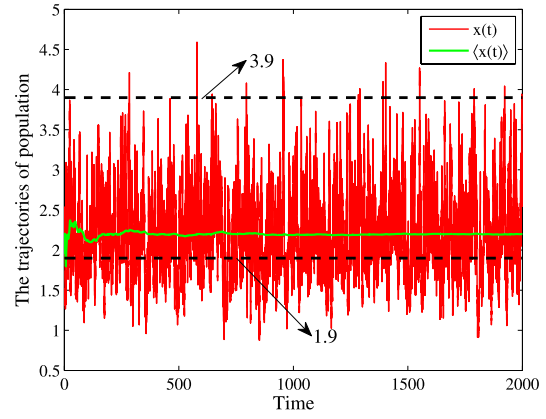

(b) Trajectories of model (2).

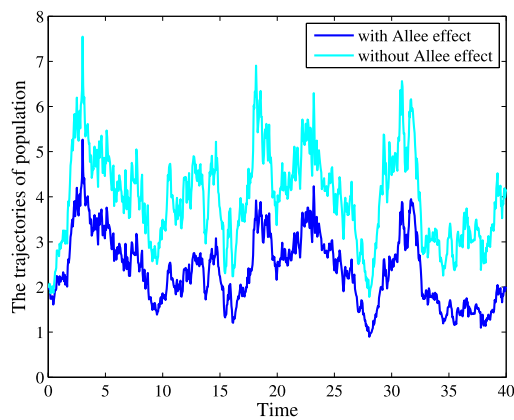

(d) Trajectories of model (3).

Figure 4. (a) trajectories of model (1.1), (b) trajectories of model (1.2), (c) trajectories of model (1.3), (d) trajectories of model (1.3) with Allee effect and without Allee effect.

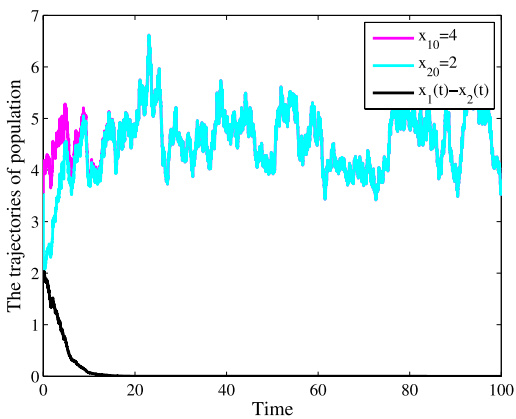

(a) Global attractivity of model (2).

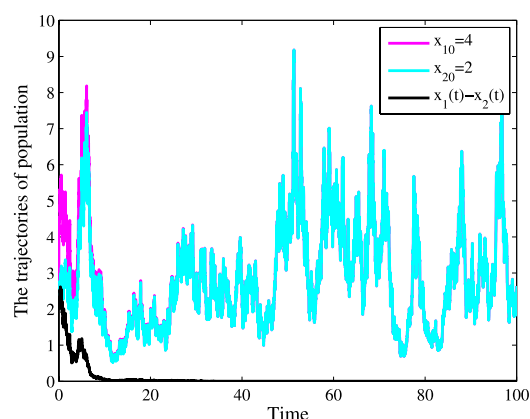

(b) Global attractivity of model (3).

Figure 5. (a) Trajectories of model (1.2) with initial values $x_{10}=4$ and $x_{20}=2$, (b) trajectories of model (1.3) with initial values $x_{10}=4$ and $x_{20}=2$, respectively.

It can be seen from Figure 5a that the solutions of model (1.2) with initial values $x_{10}=4$ and $x_{20}=2$ eventually converge to the same solution. The same result for model (1.3) can be obtained from Figure 5b.

Example 6.5. In Figure 6, choose $r=0.8, c=0.1, a=0.5, h=0.04, \sigma=0.1$ and $\gamma(z)=0.1$. Thus, $0.01=$ $h a^{2}<c=0.1$. From Theorem 5.8, it follows that model (1.3) is stable in distribution. Note that $a<r$. Then from Lemma 2.1 in [10], model (1.1) has equilibrium $x^{*}=3.3105$. 


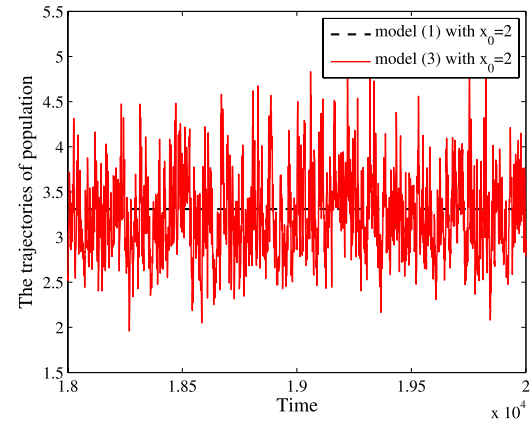

(a) Trajectories of (3) with $x_{0}=2$.

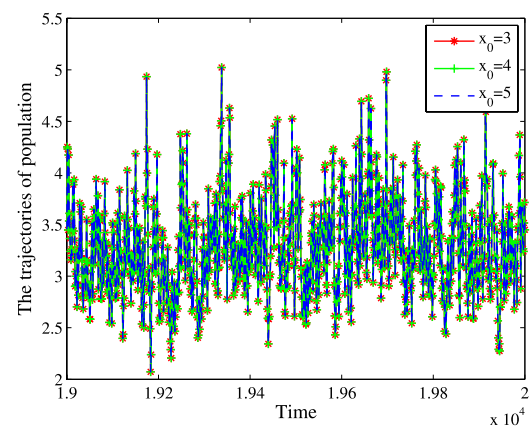

(c) Trajectories of (3) with $x_{0}=3,4,5$.

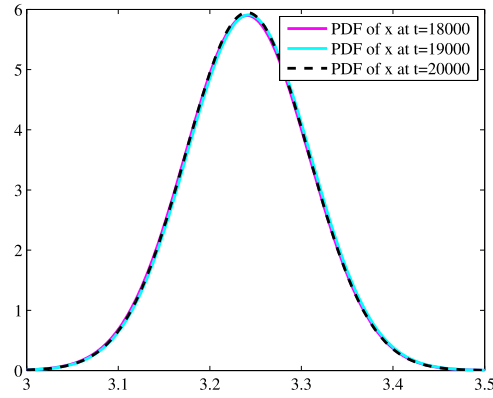

(b) PDFs of $x(t)$ at different times.

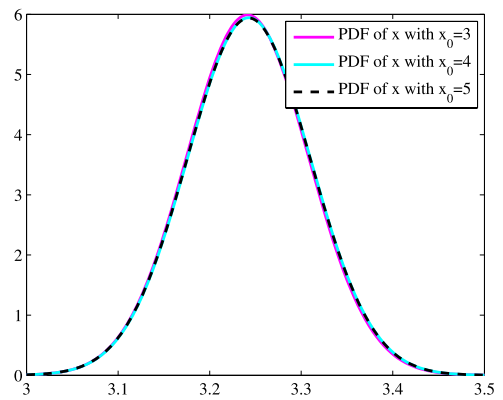

(d) PDFs of $x(t)$ with different $x_{0}$.

FigURE 6. Stochastic trajectories of(1.3) and the probability density functions (PDFs) of $x(t)$ of (1.3). (a) trajectories of (1.3) with $x_{0}=2$, (b) the PDFs of $x(t)$ at time 18000, 19000 and 20000 with $x_{0}=2,(\mathrm{c})$ trajectories of $(1.3)$ with $x_{0}=3,4,5,(\mathrm{~d})$ the PDFs of $x(t)$ at $t=20000$ with $x_{0}=3,4,5$.

From Figure $6 \mathrm{~b}$ and $\mathrm{d}$, when the time is large enough, the PDFs of (1.3) at the different times have almost no change, and PDFs of (1.3) with different initial values also have almost no change.

Example 6.6. Choose $x_{0}=2, r=0.8, c=0.2, a=0.4, h=0.2$. (i) Take $\gamma(z)=0.2$, if we choose $\sigma=0.4$, then $r+\beta-a>0$. Moreover, if we choose $\sigma=0.1(0.2,0.3)$, then $r+\beta-a>0$. This means that in the cade of $\gamma(z)=0.2$, the population described by (1.3) will be persistent in mean for different noise intensities (see Fig. 7a). (ii) Take $\sigma=0.2$, if we choose $\gamma(z)=0.4$, then $r+\beta-a>0$. Moreover, if we choose $\gamma(z)=0.1$ $(0.2,0.3)$, then $r+\beta-a>0$. This means that in the cade of $\sigma=0.2$, the population described by (1.3) will be persistent in mean for different jumps (see Fig. 7b).

It can be seen from Figure 7 that on the premise of the population is persistent in mean, the population size decreases with the increase of white noise intensity; with the increase of jump range, the population size also decreased. This means that white noise and jump are disadvantage for the survival of biological population.

\section{CONCLUding REMARKS}

This paper investigates the dynamics of a stochastic single population model with Allee effect and jumps. First, by the comparison theorem of stochastic differential equations, we show the global existence of almost surely positive solution to the model. Then, the asymptotic pathwise behavior of the model is given via the generalized exponential martingale inequality and Borel-Cantelli lemma. Meanwhile, we show that the model is stochastically ultimate bounded under some conditions by using the Itô formula and Chebyshev's inequality. 


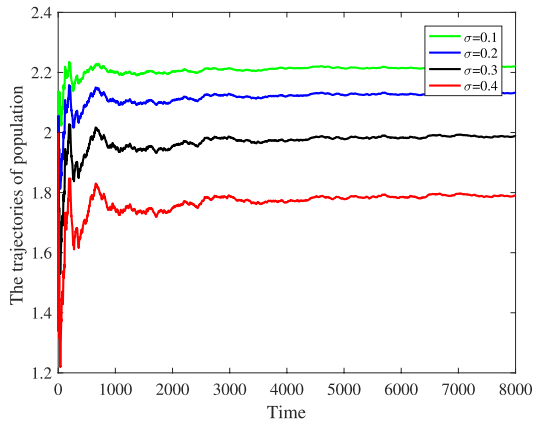

(a) Trajectories of (3) with different noise intensities.

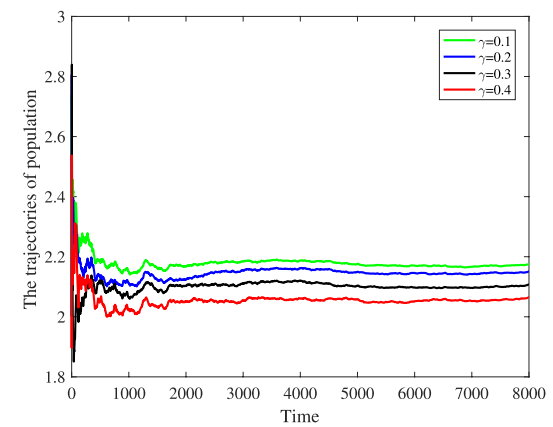

(b) Trajectories of (3) with different jumps.

FiguRE 7. (a) trajectories of model (1.3) with different noise intensities, (b) trajectories of model (1.3) with different jumps.

TABLE 1. The dynamic behavior of model (1.2) and model (1.3).

\begin{tabular}{|c|c|c|c|}
\hline & Conditions & $x(t)$ in model $(1.2)$ & $x(t)$ in model (1.3) \\
\hline \multirow{4}{*}{$a \leq \sqrt{\frac{c}{h}}$} & $r<a$ & $\lim _{t \rightarrow \infty} x(t)=0$ a.s. & $\lim _{t \rightarrow \infty} x(t)=0$ a.s. \\
\hline & $a<r<a+\frac{\sigma^{2}}{2}$ & $\lim _{t \rightarrow \infty} x(t)=0$ a.s. & $\lim _{t \rightarrow \infty} x(t)=0$ a.s. \\
\hline & $a+\frac{\sigma^{2}}{2}<r<a-\beta$ & $\liminf _{t \rightarrow \infty}\langle x(t)\rangle \geq \frac{r-\frac{\sigma^{2}}{2}-a}{c}$ a.s. & $\lim _{t \rightarrow \infty} x(t)=0$ a.s. \\
\hline & $r>a-\beta$ & $\liminf _{t \rightarrow \infty}\langle x(t)\rangle \geq \frac{r-\frac{\sigma^{2}}{2}-a}{c}$ a.s. & $\liminf _{t \rightarrow \infty}\langle x(t)\rangle \geq \frac{r+\beta-a}{c}$ a.s. \\
\hline \multirow{4}{*}{$a>\sqrt{\frac{c}{h}}$} & $0<a<C$ & Not sure & Not sure \\
\hline & $0<C<a<B$ & Not sure & $\lim _{t \rightarrow \infty} x(t)=0$ a.s. \\
\hline & $0<B<a<A$ & $\lim _{t \rightarrow \infty} x(t)=0$ a.s. & $\lim _{t \rightarrow \infty} x(t)=0$ a.s. \\
\hline & $0<A<a$ & $\lim _{t \rightarrow \infty} x(t)=0$ a.s. & $\lim _{t \rightarrow \infty} x(t)=0$ a.s. \\
\hline
\end{tabular}

Next, exponential extinction and persistence in mean are discussed. Results show that White noise, Lévy jumps and Allee effect are unfavorable for the persistence of the population. The conditions for exponential extinction in this paper generalize and improve the previous related results. Further, we show that the solution of the model is globally attractive in mean via the stochastic comparison theorem and Itô formula. At the same time, we show that the model is stable in distribution. At last, we give some numerical results to justify the analytical results.

Note that $\beta \leq-\frac{\sigma^{2}}{2}<0$. Thus, $r+\beta<r-\frac{\sigma^{2}}{2}<r$. This means that $C<B<A$. Therefore, if $r<a \leq \sqrt{\frac{c}{h}}$, then $r-\frac{\sigma^{2}}{2}<a \leq \sqrt{\frac{c}{h}}$ and $r+\beta<a \leq \sqrt{\frac{c}{h}}$. This means that if the attack rate $a$ is in intermediate range (i.e. $r<a<\sqrt{\frac{c}{h}}$ ), the populations in models (1.2) and (1.3) will go extinct regardless of the noise intensity or the jump size. Moreover, under the conditions $a>\sqrt{\frac{c}{h}}$ and $a>A>0$, if the noise intensity or jump is relatively large such that $C>0$, then the populations in models (1.2) and (1.3) will go to extinction. Further, under the condition $a<r$, if noise intensity $\sigma^{2}$ is large (i.e. $r<a+\frac{\sigma^{2}}{2}$ ), the populations in models (1.2) and (1.3) will become extinct; on the contrary, the population in model (1.2) will be persistent in mean. Further, under the condition of $a+\frac{\sigma^{2}}{2}<r$, if the jumps is large (i.e. $r<a-\beta$ ), the population in model (1.3) will become extinct; otherwise, the population in model (1.3) will be persistent in mean. Therefore, the dynamic behavior of model (1.2) and model (1.3) can be summarized as the following Table 1. 
TABLE 2. The dynamic behavior of model (1.2) and model (1.3).

\begin{tabular}{cccc}
\hline Conditions & $x(t)$ in model $(1.2)$ & $x(t)$ in model (1.3) \\
\hline & $0<r-\frac{\sigma^{2}}{2}-a$ & $\liminf _{t \rightarrow \infty}\langle x(t)\rangle \geq \frac{r-\frac{\sigma^{2}}{2}-a}{c}$ a.s. & Not sure \\
& $r-\frac{\sigma^{2}}{2}-a<0<r-\frac{\sigma^{2}}{2}+\frac{c}{h a}-2 \sqrt{\frac{c}{h}}$ & Not sure & Not sure \\
$r-\frac{\sigma^{2}}{2}+\frac{c}{h a}-2 \sqrt{\frac{c}{h}}<0$ & $\lim _{t \rightarrow \infty} x(t)=0$ a.s. & $\lim _{t \rightarrow \infty} x(t)=0$ a.s. \\
& $0<r+\beta-a$ & $\liminf _{t \rightarrow \infty}\langle x(t)\rangle \geq \frac{r-\frac{\sigma^{2}}{2}-a}{c}$ a.s. & $\liminf _{t \rightarrow \infty}\langle x(t)\rangle \geq \frac{r+\beta-a}{c}$ a.s. \\
& $r+\beta-a<0<r+\beta+\frac{c}{h a}-2 \sqrt{\frac{c}{h}}$ & Not sure & Not sure \\
& $r+\beta+\frac{c}{h a}-2 \sqrt{\frac{c}{h}}<0$ & Not sure & $\lim _{t \rightarrow \infty} x(t)=0$ a.s. \\
\hline
\end{tabular}

TABLE 3. The dynamic behavior of model (1.2) and model (1.3).

\begin{tabular}{ccc}
\hline & $r-\frac{\sigma^{2}}{2}<0$ & $\lim _{t \rightarrow \infty} x(t)=0$ a.s. \\
$x(t)$ in model (1.2) & $0<r-\frac{\sigma^{2}}{2}<a$ & Not sure \\
& $r-\frac{\sigma^{2}}{2}>a$ & $\liminf _{t \rightarrow \infty}\langle x(t)\rangle \geq \frac{r-\frac{\sigma^{2}}{2}-a}{c}$ a.s. \\
\hline & $r+\beta<0$ & $\lim _{t \rightarrow \infty} x(t)=0$ a.s. \\
$x(t)$ in model (1.3) & $0<r+\beta<a$ & Not sure \\
& $r+\beta>a$ & $\liminf _{t \rightarrow \infty}\langle x(t)\rangle \geq \frac{r-\frac{\sigma^{2}}{2}-a}{c}$ a.s. \\
\hline
\end{tabular}

If $a>\sqrt{\frac{c}{h}}$, then $-a<\frac{c}{h a}-2 \sqrt{\frac{c}{h}}<-\sqrt{\frac{c}{h}}$. Thus, we have

$$
r-\frac{\sigma^{2}}{2}-a<r-\frac{\sigma^{2}}{2}+\frac{c}{h a}-2 \sqrt{\frac{c}{h}} \text { and } r+\beta-a<r+\beta+\frac{c}{h a}-2 \sqrt{\frac{c}{h}} .
$$

From Theorems 4.1 and 4.9, we have the following results. For (1.2), if noise intensity is large such that $r-\frac{\sigma^{2}}{2}+\frac{c}{h a}-2 \sqrt{\frac{c}{h}}<0$, then the population in (1.2) will become extinct. However, if noise intensity is relatively small such that $r-\frac{\sigma^{2}}{2}-a>0$, then the population in (1.2) will be persistent in mean. For model (1.3), if noise intensity or jump is large such that $r+\beta+\frac{c}{h a}-2 \sqrt{\frac{c}{h}}<0$, then the population in (1.3) will become extinct. However, if noise intensity or jump is relatively small such that $r+\beta-a>0$, then the population in (1.3) will be persistent in mean. According to the above analysis, if the population $x(t)$ in (1.2) is extinct, the population $x(t)$ in (1.3) will become extinct. Conversely, if the population $x(t)$ in (1.3) is persistent in mean, the population $x(t)$ will be persistent in mean. Based on this, the dynamic behavior of models (1.2) and (1.3) can be summarized as the following Table 2 .

Moreover, according to Corollary 4.2 and Theorem 4.9, no matter how large or small the attack rate $a$ is, the dynamic behavior of models (1.2) and (1.3) can be seen in the following Table 3.

The results in this paper show that

- if attack rate $a$ is in the intermediate range $\left(r<a<\sqrt{\frac{c}{h}}\right)$ or very large $\left(a>\sqrt{\frac{c}{h}}\right.$ and $\left.a>\frac{c}{h} \frac{1}{2 \sqrt{\frac{c}{h}-r}}>0\right)$, the population will go extinct;

- if the attack rate $a$ is relatively small $\left(a<\sqrt{\frac{c}{h}}\right)$, the solution of the model is globally attractive in mean and the model is stable in distribution;

- under some conditions, if the noise intensity or jump is relatively large, the population will become extinct; on the contrary, the population will be persistent in mean; 
- if the attack rate $a$ is relatively small $\left(a \leq \sqrt{\frac{c}{h}}\right)$, then conditions in Theorems 4.1 and 4.9 provide a threshold condition for the development of the population in model (1.3), which depends on

$$
\mathcal{R}_{s} \doteq \frac{1}{a}[r+\beta]
$$

Roughly speaking, if $\mathcal{R}_{s}<1$, then the population in model (1.3) becomes extinct exponentially with probability one; otherwise, the population in model (1.3) is persistent in mean;

- if the population in model (1.2) is extinct, the population in model (1.3) must go to extinction. If the population in model (1.3) is persistent in mean, the population in model (1.3) must also be persistent in mean;

- the Allee effect, white noise and jump may have great influence on the survival of the population. As a result, it is more nature and realistic to use the stochastic model especially with Allee effect and jump to describing the population dynamics.

From Remark 4.5, we know that Theorem 4.1 generalizes and improves Lemma 2.1 in [10]. Moreover, from Remark 4.6, if there is only one state in the state space $\mathcal{M}$ and $c=\frac{r}{K}$ for model in [26], then extinction conditions in Theorem 4.1 are more general than those in [26].

Note that the population's environment may be randomly switched between two or more environmental regimes. As done in [26], one can introduce the Markovian switching into the stochastic population model (1.3). This is a significant problem. We leave it to future consideration.

Acknowledgements. This research was supported by the National Natural Science Foundation of China (No. 12001341, 11971279), the Youth Natural Science Foundation of Shanxi Province (No. 201901D211410) and Scientific and Technological Innovation Programs of Higher Education Institutions in Shanxi (No. 2020L0258).

\section{REFERENCES}

[1] W. Allee, Animal Aggregations. A study in General Sociology. University of Chicago Press, Chicago (1931).

[2] J. Bao, X. Mao, G. Yin and C. Yuan, Competitive Lotka-Volterra population dynamics with jumps. Nonlinear Anal. 74 (2011) 6601-6616.

[3] I. Barbalat, Systems d'equations differentisl d'oscillations nonlinearies. Rev. Roum. Math. Pures Appl. 4 (1959) $267-270$.

[4] S. Biswas, Md. Saifuddin, S. Sasmal, S. Samanta, N. Pal, F. Ababneh and J. Chattopadhyay, A delayed prey-predator system with prey subject to the strong Allee effect and disease. Nonlinear Dyn. 84 (2016) 1569-1594.

[5] D. Boukal and L. Berec, Single-species models of the Allee effect: extinction boundaries, sex ratios and mate encounters. J. Theor. Biol. 218 (2002) 375-394.

[6] J. Cushing, Backward bifurcations and strong Allee effects in matrix models for the dynamics of structured populations. J. Biol. Dynam. 8 (2014) 57-73.

[7] J. Cushing, The evolutionary dynamics of a population model with a strong Allee effect. Math. Biosci. Eng. 12 (2015) 643-660.

[8] M. Jovanović and M. Krstić, Extinction in stochastic predator-prey population model with Allee effect on prey. Discret. Contin. Dyn. Syst. Ser. B 22 (2017) 2651-2667.

[9] M. Jovanović and M. Krstić, The influence of time-dependent delay on behavior of stochastic population model with the Allee effect. Appl. Math. Model. 39 (2015) 733-746.

[10] Y. Kang and O. Udiani, Dynamics of a single species evolutionary model with Allee effects. J. Math. Anal. Appl. 418 (2014) 492-515.

[11] M. Krstić and M. Jovanović, On stochastic population model with the Allee effect. Math. Comput. Model. 52 (2010) 370-379.

[12] X. Li, A. Alison, D. Jiang and X. Mao, Sufficient and necessary conditions of stochastic permanence and extinction for stochastic logistic populations under regimes witching. J. Math. Anal. Appl. 376 (2011) 11-28.

[13] X. Li, D. Jiang and X. Mao, Population dynamical behavior of Lotka-Volterra system under regime switching. J. Comput. Appl. Math. 232 (2009) 427-448.

[14] D. Li and Y. Yang, Impact of time delay on population model with Allee effect. Commun. Nonlinear Sci. Numer. Simulat. 72 (2019) 282-293.

[15] R. Lipster, A strong law of large numbers for local martingales. Stochastics 3 (1980) 217-228.

[16] Q. Liu and Q. Chen, Asymptotic behavior of a stochastic non-autonomous predator-prey system with jumps. Appl. Math. Comput. 271 (2015) 418-428. 
[17] Q. Liu, D. Jiang, T. Hayat and A. Alsaedi, Dynamical behavior of a hybrid switching SIS epidemic model with vaccination and Lévy jumps. Stoch. Anal. Appl. 37(3) (2019) 388-411.

[18] M. Liu and K. Wang, Dynamics of a Leslie-Gower Holling-type II predator-prey system with Lévy jumps. Nonlinear Anal. 85 (2013) 204-213.

[19] X. Mao, Stochsatic Differential Equations and Applications. Horwood Publishing Limited, Chichester (2007).

[20] S. Peng and X. Zhu, Necessary and sufficient condition for comparison theorem of 1-dimensional stochastic differential equations. Stoch. Proc. Appl. 116 (2006) 370-380.

[21] J. Ripa and P. Lunddberg, Noise colour and the risk of population extinctions. P. Roy. Soc. B Biol. Sci. 263 (1996) $1751-1753$.

[22] S. Sun, Y. Sun, G. Zhang and X. Liu, Dynamical behavior of a stochastic two-species Monod competition chemostat model. Appl. Math. Comput. 298 (2017) 153-170.

[23] L. Tan, W. Jin and Y. Suo, Stability in distribution of neutral stochastic functional differential equations. Stat. Probabil. Lett. 107 (2015) 27-36.

[24] R. Wu and K. Wang, Population dynamical behaviors of stochastic logistic system with jumps. Turk. J. Math. 38 (2014) 935-948.

[25] Q. Yang and D. Jiang, A note on asymptotic behaviors of stochastic population model with Allee effect. Appl. Math. Model. 35 (2011) 4611-4619.

[26] X. Yu, S. Yuan, T. Zhang, Persistence and ergodicity of a stochastic single species model with Allee effect under regime switching. Commun. Nonlinear Sci. Numer. Simulat. 59 (2018) 359-374.

[27] Y. Zhao and S. Yuan, Stability in distribution of a stochastic hybrid competitive Lotka-Volterra model with Lévy jumps. Chaos Soliton. Fract. 85 (2016) 98-109.

[28] Y. Zhao, S. Yuan and Q. Zhang, The effect of Lévy noise on the survival of a stochastic competitive model in an impulsive polluted environment. Appl. Math. Model. 40 (2016) 7583-7600.

[29] Q. Zhang, D. Jiang, Y. Zhao and D. O'Regan, Asymptotic behavior of a stochastic population model with Allee effect by Lévy jumps. Nonlinear Anal. Hybri. 24 (2017) 1-12.

[30] B. Zhang, H. Wang and G. Lv, Exponential extinction of a stochastic predator-prey model with Allee effect. Physica A 507 (2018) 192-204.

[31] S. Zhang, T. Zhang and S. Yuan, Dynamics of a stochastic predator-prey model with habitat complexity and prey aggregation. Ecol. Complexity 45 (2021) 100889.

[32] Q. Zhu, Asymptotic stability in the pth moment for stochastic differential equations with Lévy noise. J. Math. Anal. Appl. 416 (2014) 126-142.

[33] X. Zou and K. Wang, Numerical simulations and modeling for stochastic biological systems with jumps. Commun. Nonlin. Sci. Numer. Simul. 19 (2014) 1557-1568.

[34] J. Zu and M. Mimura, The impact of Allee effect on a predator-prey system with Holling type II functional response. Appl. Math. Comput. 217 (2010) 3542-3556.

\section{Subscribe to Open (S2O) A fair and sustainable open access model}

This journal is currently published in open access under a Subscribe-to-Open model (S2O). S2O is a transformative model that aims to move subscription journals to open access. Open access is the free, immediate, online availability of research articles combined with the rights to use these articles fully in the digital environment. We are thankful to our subscribers and sponsors for making it possible to publish this journal in open access, free of charge for authors.

\section{Please help to maintain this journal in open access!}

Check that your library subscribes to the journal, or make a personal donation to the $\mathrm{S} 2 \mathrm{O}$ programme, by contacting subscribers@edpsciences.org

More information, including a list of sponsors and a financial transparency report, available at: https://www.edpsciences.org/en/maths-s2o-programme 